\title{
A NOTE ON THE SECOND VARIATIONAL FORMULAS OF FUNCTIONALS ON RIEMANN SURFACES
}

\author{
By Masahiko TANiguchi
}

\section{§1. Introduction.}

Recently, F. Maitani proved the second variational formulas for several fundamental functionals of arbitrary Riemann surfaces when surfaces varies holomorphically $([6, \S 3])$. His proof based on the classical and fundamental method of orthogonal decomposition of square integrable abelian differentials. But since he considered very general classes of differentials, the key of his argument is not so clear. Hence it seems helpful to give a short and rather elementary proof in case of typical and fundamental functionals, such as the extremal length of the homology class of a given curve and Robin's constant at a given point (which were originally investigated by $\mathrm{H}$. Yamaguchi [9]).

In this paper, the author uses only two very elementary orthogonal decompositions. But we succeed in handling fairly general quasiconformal deformations and giving a general second variational formulas which reduces to Maitani's ones in case of holomorphic families of surfaces.

In the next section, we will state the second variational formulas for the above two functionals under general quasiconformal deformation. Proofs of formulas, which the author intends to be self-contained, will be given in $\$ 4$ after preparing more general second variational formulas in $\S 3$. The lines of the proofs are similar to those of Maitani's ones.

\section{$\S 2$. Statements of main results.}

1. In general, for a given family $\{x(t) \in X: t \in I, I$ is a neighborhood of 0 in $\Re\}$ of elements in a Banach space $X$ with the norm $\|\cdot\|_{X}$, we say that $x(t)$ is differentiable at $t_{0} \in I$ if there is an element $y \in X$ such that $\lim _{t \neq 0, t \rightarrow 0} 1 / t$. $\left\|\left(x\left(t+t_{0}\right)-x\left(t_{0}\right)\right)-t y\right\|_{X}=0$, and denote $y$ by $(d x / d t)\left(t_{0}\right)$. When $x(t)$ depends on several real parameters $t=\left(t_{1}, \cdots, t_{n}\right)$, then, for every $j$, partial differentiability and the partial derivative $\partial x / \partial t$, are defined in a similar way (cf. [5, Ch. 8]).

For a Riemann surface $R$, we denote by $\Gamma(R)$ the Hilbert space consisting of all real square integrable abelian differentials on $R$ with the inner product

Received April 14, 1988. 
$(\alpha, \beta)_{R}=\iint_{R} \alpha \wedge^{*} \beta$. Let $\Gamma_{c}(R)$ and $\Gamma_{h}(R)$ be subspaces of $\Gamma(R)$ consisting of all closed differentials, and of all harmonic ones, respectively. We denote by $\Gamma_{e 0}(R)$ the closure of $\{d f: f$ is smooth and has a compact support in $R\}$ in $\Gamma(R)$. Then the following orthogonal decompositions are classically well-known (cf. [3, Ch. V, §2]);

$$
\Gamma(R)=\Gamma_{c}(R)+* \Gamma_{e 0}(R), \quad \text { and } \quad \Gamma_{c}(R)=\Gamma_{h}(R)+\Gamma_{e 0}(R),
$$

where we set $* \Gamma_{e 0}(R)=\left\{* d f: d f \in \Gamma_{e 0}(R)\right\}$. Next for the complex Hilbert space $\Gamma^{\Subset}(R)=\{\alpha+i \beta: \alpha, \beta \in \Gamma(R)\}$ with the inner product $(\varphi, \phi)_{R}^{\pi}=\iint_{R} \varphi \wedge^{*} \bar{\psi}$, the following orthogonal decomposition is clear;

$$
\Gamma^{\natural}(R)=\Gamma^{1,0}(R)+\Gamma^{0,1}(R),
$$

where we set $\Gamma^{1,0}(R)=\left\{\phi \in \Gamma^{\circledR}(R):{ }^{*} \phi=-i \phi\right\}$ and $\Gamma^{0,1}(R)=\left\{\phi \in \Gamma^{\mathbb{E}}(R):{ }^{*} \phi=\right.$ $i \phi\}$. Also we set $\Gamma_{\chi}^{\S}(R)=\left\{\alpha+i \beta: \alpha, \beta \in \Gamma_{\chi}(R)\right\}$ with $\chi=c, h$ or $e 0$.

2. Let $R$ be an arbitrary Riemann surface, and $B(R)$ be the complex Banach space consisting of all Beltrami differentials, i.e. all bounded $(-1,1)$-forms, $\mu$ on $R$ with the norm $\|\mu\|_{\infty}=$ ess. $\sup _{p \in R}|\mu|(p)$. First we consider a real 1-parameter family $\{\mu(t): t \in I\}$ in $B(R)$, and suppose that

$$
\begin{aligned}
& \mu(0) \equiv 0 \text { and }\|\mu(t)\|_{\infty}<1 \text { for every } t, \text { and } \\
& \mu(t) \text { is differentiable at every } t \in I .
\end{aligned}
$$

Let $f_{t}$ be the quasiconformal mapping of $R=R_{0}$ to another $R_{t}$ with the complex dilatation $\mu(t)$ for every $t$. In the sequel, we denote by $\alpha_{t} \circ f_{t}$ the pull-back of a differential $\alpha_{t}$ on $R_{t}$ by $f_{t}$.

3. Fix a closed curve $C_{0}$ on $R_{0}$ and let $C_{t}$ be the 1-cycle on $R_{t}$ determined by the curve $f_{t}\left(C_{0}\right)$. Let $\sigma_{t}=\sigma\left(C_{t}, R_{t}\right)$ be the period reproducer of $C_{t}$ in $\Gamma_{h}\left(R_{t}\right)$, i.e. the differential in $\Gamma_{h}\left(R_{t}\right)$ such that

$$
\left(\alpha, \sigma_{t}\right)_{R_{t}}=\int_{C_{t}} \alpha \quad \text { for every } \alpha \in \Gamma_{h}\left(R_{t}\right),
$$

and $\lambda(t)$ be the extremal length of the homology class of $C_{t}$. Then Accola's theorem ([1]) implies $\lambda(t)=\left\|\sigma_{t}\right\|_{R_{t}}^{2}$, and we have the following

THEOREM 1. Under the assumptions (i) and (ii), further suppose that

$$
\frac{d \mu}{d t} \text { is differentiable at } t=0 \text {. }
$$

Then $\lambda(t)$ is twice differentiable at $t=0$. 
Moreover, set $\phi_{t}=\sigma_{t}+i \cdot * \sigma_{t}$ and $\Phi(t)=\phi_{t} \circ f_{t}-\phi_{0}$ for every $t$. Then $\Phi(t)$ is differentiable (in $\left.\Gamma\left(R_{0}\right)\right)$ at $t=0$ and

$$
\frac{d^{2} \lambda}{d t^{2}}(0)=\operatorname{Re} \iint_{R_{0}}\left(\frac{d^{2} \mu}{d t^{2}}(0) \phi_{0} \wedge^{*} \phi_{0}+\frac{d \Phi}{d t}(0) \wedge^{*} \frac{d \Phi}{d t}(0)\right)
$$

4. Next fix a point $p_{0}$ on $R_{0}$, and suppose that $R_{0}$ admits Green's functions. Fix a simply connected neighborhood $U_{0}$ of $p_{0}$ in $R_{0}$ and a conformal mapping $Z_{0}$ of $U_{0}$ onto the unit disk $B=\{|z|<1\}$ such that $Z_{0}\left(p_{0}\right)=0$. Assume that

$$
\mu(t) \equiv 0 \quad \text { on } U_{0} \quad \text { for every } t .
$$

Then $Z_{t}=Z_{0^{\circ}}\left(f_{t}\right)^{-1}$ is a conformal mapping of $f_{t}\left(U_{0}\right)$ onto $B$ such that $Z_{t}\left(p_{t}\right)$ $=0$, where $p_{t}=f_{t}\left(p_{0}\right)$.

Let $g_{t}(p)=g\left(p, p_{t} ; R_{t}\right)$ be Green's function on $R_{t}$ with the pole $p_{t}$ for every $t$. Then we can define Robin's constant $\gamma(t)$ at $p_{t}$ on $R_{t}$ by setting

$$
\gamma(t)=\lim _{z \rightarrow 0} g_{t}\left(Z_{t}^{-1}(z)\right)+\log |z|
$$

for every $t$, and we have the following

THEOREM 1'. Under the assumptions (i) and (ii), further suppose that $\{\mu(t)\}$ satisfies (iii) and (iv). Then $\gamma(t)$ is twice differentiable at $t=0$.

Moreover, set $\phi_{t}=-* d g_{t}+i \cdot d g_{t}$ and $\Phi(t)=\phi_{t} \circ f_{t}-\phi_{0}$. Then $\Phi(t)$ is differentiable at $t=0$ and

$$
\frac{d^{2} \gamma}{d t^{2}}(0)=\frac{-1}{2 \pi} \cdot \operatorname{Re} \iint_{R_{0}}\left(\frac{d^{2} \mu}{d t^{2}}(0) \phi_{0} \wedge^{*} \phi_{0}+\frac{d \Phi}{d t}(0) \wedge^{*} \frac{d \Phi}{d t}(0)\right) .
$$

5. Now we consider the case that $\mu$ depends on a complex parameter $t$ on a neighborhood $U$ of 0 in $(5$. Then considering that $\mu(t)$ depends on two real parameters $x=\operatorname{Re} t$ and $y=\operatorname{Im} t$, we write $\mu(x+i y)$ also by $\mu(x, y)$.

Suppose that $\{\mu(t)\}$ satisfies (i) and that

$$
\frac{\partial \mu}{\partial x} \text { and } \frac{\partial \mu}{\partial y} \text { exist at every }(x, y) \text { with } x+i y \in U \text {. }
$$
lowing

Let $\left\{f_{t}\right\}$ and $\left\{R_{t}\right\}$ be as in $\S 2-2$. Then Theorems 1 and $1^{\prime}$ imply the fol-

THEOREM 2 (cf. $[6, \S 3]$ ). Under the assumptions (i) and (ii'), further sufpose that

$$
\begin{aligned}
& \frac{\partial^{2} \mu}{\partial x^{2}} \text { and } \frac{\partial^{2} \mu}{\partial y^{2}} \text { exist at } t=0 \text {, and } \\
& \frac{\partial \mu}{\partial \bar{t}}(0)=0, \text { and } \Delta \mu(0)=0 \text {. }
\end{aligned}
$$


Then

$$
\Delta \lambda(0)=4 \cdot\left\|\frac{\partial \Phi}{\partial \bar{t}}(0)\right\|_{R_{0}}^{2} \geqq 0
$$

Moreover if $\{\mu(t)\}$ also satisfies (iv), then

$$
\Delta \gamma(0)=-\frac{2}{\pi} \cdot\left\|\frac{\partial \Phi}{\partial t}(0)\right\|_{R_{0}}^{2} \leqq 0,
$$

where we set $\partial / \partial t=1 / 2((\partial / \partial x)-i \cdot \partial / \partial y), \partial / \partial \bar{t}=1 / 2((\partial / \partial x)+i \cdot \partial / \partial y)$, and $\Delta=$ $\left(\left(\partial^{2} / \partial x^{2}\right)+\partial^{2} / \partial y^{2}\right)$ as usual.

Now it is easy to show the following

Corollary ([6], [9]). Suppose that $\mu$ depends on $n$ complex parameters $t=$ $\left(t_{1}, \cdots, t_{n}\right)$ holomorphically on a neighborhood $U$ of $(0, \cdots, 0)$ in $\mathfrak{S}^{n}$, and that (i) in $\S 2-2$ holds. Then $\lambda(t)$ is continuous and plurisubharmonic on $U$.

Further if $\{\mu\}$ also satisfies (iv), then $\gamma(t)$ is continuous and plurisuperharmonic on $U$.

Here recall that one of mutually equivalent definitions of holomorphical dependence of $\mu$ on $t$ is the following (cf., for instance, [5, 8.9 and 9.10]);

$$
\mu \text { and all } \frac{\partial \mu}{\partial\left(\operatorname{Re} t_{j}\right)}, \frac{\partial \mu}{\partial\left(\operatorname{Im} t_{j}\right)} \quad(j=1, \cdots, n)
$$

are (exist and) continuous on $U$, and

$$
\frac{\partial \mu}{\partial \bar{t}_{\jmath}} \equiv 0 \quad \text { on } U \text { for every } j \text {. }
$$

\section{§ 3. A general second variational formula.}

For the sake of convenience, we include proofs of all lemmas in this section, though some of them are well-known.

1. Let $\{\mu(t)\},\left\{f_{t}\right\}$ and $\left\{R_{t}\right\}$ be as in $\S 2-2$, and a meromorphic differential $\phi_{t}$ on $R_{t}$ be given for every $t$. Set $\Phi(t)=\phi_{t} \circ f_{t}-\phi_{0}$, and assume that

$\left(\mathrm{A}^{0}\right) \quad \omega(t)=\operatorname{Re} \Phi(t) \in \Gamma_{c}\left(R_{0}\right)$, and $\tau(t)=\operatorname{Im} \Phi(t) \in \Gamma_{e 0}\left(R_{0}\right)$ for every $t$, and

$\left(\mathrm{B}^{0}\right) \quad$ there is a subsurface $S$ of $R_{0}$ such that $\left\|\phi_{0}\right\|_{S}<+\infty$ and

$$
\mu(t) \equiv 0 \quad \text { on } R_{0}-S \quad \text { for every } t .
$$

Consider the following elementary orthogonal decomposition of $\Phi(t)$ due to (2) in $\S 2$; 
(1)

$$
\begin{gathered}
\Phi(t)=\Phi^{1,0}(t)+\Phi^{0,1}(t) ; \\
\Phi^{1,0}(t) \in \Gamma^{1,0}\left(R_{0}\right), \quad \Phi^{0,1}(t) \in \Gamma^{0,1}\left(R_{0}\right) .
\end{gathered}
$$

Then the following two lemmas are well-known (cf., for instance, [6], [8] and References of them).

LEMMA 1. For every $t$,

$$
\Phi^{0,1}(t)=\mu(t) \cdot\left(\Phi^{1,0}(t)+\phi_{0}\right),
$$

and

$$
\left\|\Phi^{1,0}(t)\right\|_{R_{0}}=\left\|\Phi^{0,1}(t)\right\|_{R_{0}} \leqq \frac{k_{t}}{1-k_{t}} \cdot\left\|\phi_{0}\right\|_{S},
$$

where $k_{t}=\|\mu(t)\|_{\infty}<1$.

Moreover $\Phi^{0,1}(t)$ is differentiable at $t=0\left(\right.$ in $\left.\Gamma^{0,1}\left(R_{0}\right)\right)$ and

$$
\frac{d \Phi^{0,1}}{d t}(0)=\frac{d \mu}{d t}(0) \phi_{0} .
$$

Proof. First write $\phi_{t}=a_{t}\left(z_{t}\right) d z_{t}$ and $f_{t}=\left(z_{t}\right)^{-1} \circ F_{t} \circ z$ with generic local parameters $z_{t}$ and $z=z_{0}$ on $R_{t}$ and $R_{0}$, respectively. Then we have

$$
\Phi^{1,0}(t)=a_{t}\left(F_{t}(z)\right) \cdot\left(F_{t}\right)_{z} d z-\phi_{0},
$$

and

$$
\Phi^{0,1}(t)=a_{t}\left(F_{t}(z)\right) \cdot\left(F_{t}\right)_{\bar{z}} d \bar{z} .
$$

Hence we have the equation (2).

Next by $\left(A^{0}\right)$ and $(1)$ in $\S 2$, we have

$$
\begin{aligned}
N_{t} & \equiv \iint_{R_{0}} \Phi(t) \wedge \overline{\Phi(t)}=-2 i \cdot \iint_{R_{0}} \omega(t) \wedge \tau(t) \\
& =2 i \cdot\left(\omega(t),{ }^{*} \tau(t)\right)_{R_{0}}=0
\end{aligned}
$$

On the other hand,

$$
\begin{aligned}
N_{t} & =\iint_{R_{0}} \Phi^{1,0}(t) \wedge \overline{\Phi^{1,0}(t)}+\iint_{R_{0}} \Phi^{0,1}(t) \wedge \overline{\Phi^{0,1}(t)} \\
& =-i \cdot\left\|\Phi^{1,0}(t)\right\|_{R_{0}}^{2}+i \cdot\left\|\Phi^{0,1}(t)\right\|_{R_{0}}^{2} .
\end{aligned}
$$

Hence set $E_{t} \equiv\left\|\Phi^{0,1}(t)\right\|_{R_{0}}$. Then $E_{t}=\left\|\Phi^{1,0}(t)\right\|_{R_{0}}$. And since $E_{t} \leqq k_{t} \cdot\left(E_{t}+\left\|\phi_{0}\right\|_{S}\right)$ by $\left(\mathrm{B}^{0}\right)$ and (2), we conclude the inequality (3).

Finally since $\left(\Phi^{0,1}(t)-\Phi^{0,1}(0)\right) / t=(\mu(t) / t) \cdot\left(\Phi^{1,0}(t)+\phi_{0}\right)$ by $(2), \mu(t) / t$ converges to $(d \mu / d t)(0)$ in $B\left(R_{0}\right)$ by (ii), and $\Phi^{1,0}(t)$ converges to 0 in $\Gamma\left(R_{0}\right)$ by (3), we conclude the second assertion and the equation (4). q.e.d. 
LEMMA 2. Suppose that

$\left(\mathrm{C}^{0}\right) \quad \Phi(t) \wedge^{*} \phi_{0}$ is absolutely integrable on $R_{0}$ for every $t$.

And set $I(t)=\iint_{R_{0}} \Phi(t) \wedge * \phi_{0} . \quad$ Then $I(t)$ is differentiable at $t=0$ and

$$
\frac{d I}{d t}(0)=\iint_{R_{0}} \frac{d \mu}{d t}(0) \phi_{0} \wedge^{*} \phi_{0}
$$

Proof. Since ${ }^{*} \phi_{0}=-i \phi_{0},(1),(2)$ and $\left(\mathrm{B}^{0}\right)$ implies that

$$
\begin{aligned}
\frac{I(t)-I(0)}{t} & =\iint_{R_{0}}\left[\frac{\Phi(t)-\Phi(0)}{t}\right] \wedge^{*} \phi_{0} \\
& =\iint_{S}\left[\frac{\Phi^{0,1}(t)-\Phi^{0,1}(0)}{t}\right] \wedge^{*} \phi_{0} .
\end{aligned}
$$

Hence by the second assertion of Lemma $1, I(t)$ is differentiable at $t=0$, and (7) holds by (4). q.e.d.

Remark. In the above proof, we have used only the differentiability of $\mu(t)$ only at $t=0$.

Next recalling $\left(\mathrm{A}^{0}\right)$, we have

and

$$
2 \cdot \Phi^{1,0}(t)=(\omega(t)+i \cdot * \omega(t))+i \cdot(\tau(t)+i \cdot * \tau(t)),
$$

$$
2 \cdot \Phi^{0.1}(t)=(\omega(t)-i \cdot * \omega(t))+i \cdot(\tau(t)-i \cdot * \tau(t)) .
$$

And we can see the following

Lemma 3 (cf. [6, Theorem 1]). In $\Gamma^{\S}\left(R_{0}\right), \Phi(t)$ is differentiable at $t=0$, hence so is $\Phi^{1,0}(t)$.

Also $\omega(t)$ and $\tau(t)$ are differentiable at $t=0$ in $\Gamma\left(R_{0}\right)$.

Proof. By the second assertion of Lemma $1, \operatorname{Re} \Phi^{0,1}(t)=\omega(t)+^{*} \tau(t)$ is differentiable at $t=0$. Since $\omega(t) \in \Gamma_{c}\left(R_{0}\right),{ }^{*} \tau(t) \in{ }^{*} \Gamma_{e 0}\left(R_{0}\right)$, and each projection mapping is bounded linear, $\omega(t)$ and $\tau(t)$ are differentiable at $t=0$, hence so is $\Phi(t)=\omega(t)+i \cdot \tau(t)$.

q.e.d.

2. Now take another $T$ in $I$ arbitrarily and replace the center $R_{0}$ by $R_{T}$. Then the quasiconformal mapping $f_{t}^{T}=f_{t} \circ\left(f_{T}\right)^{-1}$ of $R_{T}$ to $R_{t}$ has the complex dilatation $\nu(t-T)=\nu\left(t-T ; z_{T}\right) \cdot\left(d \bar{z}_{T} / d z_{T}\right)$ with

$$
\nu\left(t-T ; z_{T}\right)=\left[\frac{\mu(t ; z)-\mu(T ; z)}{1-\overline{\mu(T ; z)} \cdot \mu(t ; z)} \cdot \frac{\left(F_{T}\right)_{z}}{\left(\bar{F}_{T}\right)_{\bar{z}}}\right] \circ\left(F_{T}\right)^{-1},
$$

where $z, z_{T}$ and $F_{T}$ are as in the proof of Lemma 1 (with $t=T$ ) and we write 
$\mu(t)=\mu(t ; z) \cdot(d \bar{z} / d z)($ cf. $[2$, Ch. 1-(10)]).

By (ii), $\mu(t)$ is differentiable at $t=T$ in $B\left(R_{0}\right)$, hence so is $\nu(s)$ at $s \equiv t-T=0$ in $B\left(R_{T}\right)$. Also the equation

$$
\frac{d \nu\left(\cdot ; z_{T}\right)}{d s}(0)=\left[\frac{\frac{d \mu(\cdot ; z)}{d t}(T)}{1-|\mu(T ; z)|^{2}} \cdot \frac{\left(F_{T}\right)_{z}}{\left(\bar{F}_{T}\right)_{\bar{z}}}\right] \circ\left(F_{T}\right)^{-1}
$$

holds. So we have the following

LEMMA 4 (cf. [6, Theorem 2]). Set $\Phi^{T}(s)=\phi_{s+T} \circ f_{s+T}^{T}-\phi_{T}$, and assume that $\left(\mathrm{A}^{T}\right) \quad \operatorname{Re} \Phi^{T}(s) \in \Gamma_{c}\left(R_{T}\right)$ and $\operatorname{Im} \Phi^{T}(s) \in \Gamma_{e 0}\left(R_{T}\right)$,

$\left(\mathrm{B}^{T}\right) \quad$ there is a subsurface $S_{T}$ of $R_{T}$ such that $\left\|\phi_{T}\right\|_{S_{T}}<+\infty$ and $\nu(s) \equiv 0$ on $R_{T}-S_{T}$, and

$\left(\mathrm{C}^{T}\right) \quad \Phi^{T}(s) \wedge{ }^{*} \phi_{T}$ is absolutely integrable on $R_{T}$

for every s with $s+T \in I$. For every such s, set

$$
I(s ; T)=\iint_{R_{T}} \Phi^{T}(s) \wedge^{*} \phi_{T} .
$$

Then $I(s ; T)$ is differentiable at $s=0$ and

$$
\begin{aligned}
\dot{I}(T) \equiv \frac{d I(\cdot ; T)}{d s}(0) & =\iint_{R_{T}} \frac{d \nu}{d s}(0) \phi_{T} \wedge^{*} \phi_{T} \\
& =\iint_{R_{0}} \frac{d \mu}{d t}(T)\left(\Phi^{1,0}(T)+\phi_{0}\right) \wedge *\left(\Phi^{1,0}(T)+\phi_{0}\right)
\end{aligned}
$$

Proof. By the same argument as in the proofs of Lemmas 1 and 2 we can show the first assertion and the first equation of (11). Next change the variable $z_{T}$ to $z$, and note that $d \nu / d s(0) \equiv 0$ on $R_{T}-S_{T}$ by $\left(B^{T}\right)$. Then we see by (9) that

$$
\begin{aligned}
& \iint_{R_{T}} \frac{d \nu}{d s}(0) \phi_{T} \wedge^{*} \phi_{T} \\
& \quad=\iint_{R_{0}} \frac{d \mu(\cdot ; z)}{d t}(T) \cdot\left(a_{T} \circ F_{T}\right) \cdot\left(F_{T}\right)_{z} d \bar{z} \wedge\left(-i \cdot a_{T} \circ F_{T}\right) \cdot\left(F_{T}\right)_{z} d z,
\end{aligned}
$$

which implies the second equation of (11) by (5).

q.e.d.

THEOREM 3 (cf. [6, Theorem 3]). Suppose that $\left(\mathrm{A}^{T}\right),\left(\mathrm{B}^{T}\right)$ and $\left(\mathrm{C}^{T}\right)$ holds for every $T \in I$, and further assume that (iii) in Theorem 1 holds. Then $\dot{I}(T)$ is differentiable at $T=0$ and 


$$
\frac{d \dot{I}}{d T}(0)=\iint_{R_{0}}\left(\frac{d^{2} \mu}{d t^{2}}(0) \phi_{0} \wedge^{*} \phi_{0}+\frac{d \Phi}{d t}(0) \wedge^{*} \frac{d \Phi}{d t}(0)\right)
$$

Proof. Since $\left\|\Phi^{1,0}(T)\right\|_{R_{0}}=O(T)$ as $T$ tends to 0 by (ii) and (3), and since $\|(d \mu / d t)(T)-(d \mu / d t)(0)\|_{\infty}=O(T)$ by (iii), $\left(\mathrm{B}^{0}\right)$ and (11) implies that

$$
\dot{I}(T)=\iint_{S}\left(\frac{d \mu}{d t}(T) \phi_{0} \wedge^{*} \phi_{0}-2 i \cdot \frac{d \mu}{d t}(0) \phi_{0} \wedge \Phi^{1,0}(T)\right)+O\left(T^{2}\right) .
$$

Hence (iii) and (4) implies

$$
\frac{d \dot{I}}{d T}(0)=\iint_{S}\left(\frac{d^{2} \mu}{d t^{2}}(0) \phi_{0} \wedge^{*} \phi_{0}-2 i \cdot \frac{d \Phi^{0,1}}{d t}(0) \wedge \frac{d \Phi^{1,0}}{d t}(0)\right) .
$$

Here set $\dot{\Phi}^{1,0}=\left(d \Phi^{1,0} / d t\right)(0), \dot{\Phi}^{0,1}=\left(d \Phi^{0,1} / d t\right)(0)$ and $\dot{\Phi}=(d \Phi / d t)(0)$. Then $\dot{\Phi}^{1,0} \in$ $\Gamma^{1,0}\left(R_{0}\right)$ and $\dot{\Phi}^{0,1} \in \Gamma^{0,1}\left(R_{0}\right)$, and hence we have

$$
\begin{gathered}
-2 i \cdot \iint_{S} \dot{\Phi}^{0,1} \wedge \dot{\Phi}^{1,0}=-i \cdot \iint_{R_{0}}\left(\dot{\Phi} \wedge \dot{\Phi}^{1,0}+\dot{\Phi}^{0,1} \wedge \dot{\Phi}\right) \\
\left.=\iint_{R_{0}} \dot{\Phi} \wedge{ }^{*} \dot{\Phi}^{1,0}+* \dot{\Phi}^{0,1}\right)=\iint_{R_{0}} \dot{\Phi} \wedge * \dot{\Phi}
\end{gathered}
$$

Thus (13) follows from (14) and (15).

q.e.d.

Remark 2. Actually, we have used $\left(\mathrm{A}^{T}\right)$ to show that $\left(\operatorname{Re} \Phi^{T}(t-T)\right.$, $\left.* \operatorname{Im} \Phi^{T}(t-T)\right)=0$ for every $t$ and $T$. Recall that the behavior conditions such as used by Maitani also assure us the desired orthogonality, by definition.

\section{§4. Proofs of Theorems 1, 2 and Corollary.}

Although all lemmas in this section are well-known, we again include direct proofs for the sake of convenience.

1. Proof of Theorem 1. Set $\phi_{t}=\sigma_{t}+i * \sigma_{t}$ and $\Phi^{T}(t)=\phi_{t} \circ f_{t}^{T}-\phi_{T}$ for every $t$ and $T$, where $f_{t}^{T}=f_{t} \circ\left(f_{T}\right)^{-1}$. Also we recall a standard construction of $\sigma_{t}$ (cf. [3, V-19]). We may assume without loss of generality that $C_{0}$ is simple, and take a doubly connected relatively compact subdomain $W$ of $R_{0}$ whose relative boundary consists of two smooth Jordan curves, say $c^{+}$and $c^{-}$, homotopic to the given $C_{0}$ and to $-C_{0}$, respectively. For every $t$, let $u_{t}$ be a bounded smooth function on $W_{t}=f_{t}(W)$ such that $u_{t} \equiv 1$ and $u_{t} \equiv 0$ in neighborhoods of $f_{t}\left(c^{+}\right)$and $f_{t}\left(c^{-}\right)$, respectively. Then we can consider $d u_{t}$ as an element of $\Gamma_{c}\left(R_{t}\right)$, by setting $d u_{t} \equiv 0$ on $R_{t}-W_{t}$. Green's formula gives

$$
\left(\omega, * d u_{t}\right)_{R_{t}}=\iint_{W_{t}} d u_{t} \wedge \omega=\int_{C_{t}} \omega=\left(\omega, \sigma_{t}\right)_{R_{t}}
$$

for every smooth $\omega \in \Gamma_{c}\left(R_{t}\right)$, which implies that $* d u_{t}-\sigma_{t} \in{ }^{*} \Gamma_{e 0}\left(R_{t}\right)$. 
LEMMA 5. The family $\left\{\Phi^{T}(s): s+T \in I\right\}$ satisfies $\left(\mathrm{A}^{T}\right),\left(\mathrm{B}^{T}\right)$ and $\left(\mathrm{C}^{T}\right)$ for every $T \in I$.

Proof. Fix $T \in I$ and take any simply connected domain $D$ on $R_{T}$. Then there is a harmonic function $v_{t}$ on $f_{t}^{T}(D)$ such that $d v_{t}=\sigma_{t}$ for every $t$. Since $\operatorname{Re} \Phi^{T}(t-T)=d\left(v_{t} \circ f_{t}^{T}-v_{T}\right)$ on $D$, it belongs to $\Gamma_{c}(D)$ (, where and in the sequel, $d u$ denotes the distributional total differential of $u$ if $u$ is not smooth). Since a locally closed differential is closed (cf. [7, Proposition 4]), we conclude that $\operatorname{Re} \Phi^{T}(t-T) \in \Gamma_{c}\left(R_{T}\right)$ for every $t$.

Next set $\alpha_{t}=d u_{t}+{ }^{*} \sigma_{t}$ and $h_{t}=u_{t} \circ f_{t}^{T}-u_{T}$ for every $t$. Then $\alpha_{t} \in \Gamma_{e 0}\left(R_{t}\right)$ as is shown above. Also since $h_{t}$ has a compact support on $W_{T}$, a generalized Green's formula ([7, Proposition 3]) gives that $\left(d h_{t}, * \omega\right)_{R_{T}}=\iint_{W_{T}}-d h_{t} \wedge \omega=$ $\int_{\partial W_{T}}-h_{t} \cdot \omega=0$ for every smooth $\omega \in \Gamma_{c}\left(R_{T}\right)$. Hence $d h_{t} \in \Gamma_{e 0}\left(R_{T}\right)$ for every $t$.

Similarly, we can show that $\alpha_{t} \circ f_{t}^{T} \in \Gamma_{e 0}\left(R_{T}\right)$. (Cf. [7, Theorem 3]. In fact, approximate $\alpha_{t}$ by $d h$ with smooth functions $h$ with compact supports. Then as above we can see that $d\left(h \circ f_{t}^{T}\right) \in \Gamma_{e 0}\left(R_{T}\right)$, and a simple estimation shows that $\left\|\left(d h-\alpha_{t}\right) \circ f_{t}^{T}\right\|_{R_{T}}^{2} \leqq K_{t}^{T} \cdot\left\|d h-\alpha_{t}\right\|_{R_{t}}^{2}\left(\left[7\right.\right.$, Theorem 2]), where $K_{t}^{T}$ is the maximal dilatation of $f_{t}^{T}$. Hence we can see the assertion.)

Thus we conclude that $\operatorname{Im} \Phi^{T}(t-T)=\left({ }^{*} \sigma_{t}\right) \circ f_{t}^{T}-{ }^{*} \sigma_{T}=\alpha_{t} \circ f_{t}^{T}-\alpha_{T}-d h_{t}$ belongs to $\Gamma_{e 0}\left(R_{T}\right)$.

Finally since $\phi_{T} \in \Gamma_{h}^{\nwarrow}\left(R_{T}\right)$, $\left(\mathrm{B}^{T}\right)$ holds with $S_{T}=R_{T}$ and also $\left(\mathrm{C}^{T}\right)$ does.

LEMMA 6. For every $t$ and $T$ in $I$, q. e. d.

$$
\lambda(t)-\lambda(T)=\operatorname{Re} \iint_{R_{T}} \Phi^{T}(t-T) \wedge * \phi_{T} .
$$

Proof. Since $\operatorname{Im} \Phi^{T}(t-T) \in \Gamma_{e 0}\left(R_{T}\right)$ by Lemma 5, $\left(\operatorname{Im} \Phi^{T}(t-T), *^{*} \sigma_{T}\right)_{R_{T}}=0$. Hence we have

$$
\begin{aligned}
\operatorname{Re} \iint_{R_{T}} \Phi^{T}(t-T) \wedge^{*} \phi_{T} & =\left(\operatorname{Re} \Phi^{T}(t-T), \sigma_{T}\right)_{R_{T}} \\
& =\left(\sigma_{t} \circ f_{t}^{T}, \sigma_{T}\right)_{R_{T}}-\left\|\sigma_{T}\right\|_{R_{T}}^{2} .
\end{aligned}
$$

Here again by Proposition 3 of [7],

$$
\begin{aligned}
\left(\sigma_{t} \circ f_{t}^{T}, \sigma_{T}\right)_{R_{T}} & =\left(\sigma_{t} \circ f_{t}^{T}, * d u_{T}\right)_{R_{T}}=-\iint_{R_{T}} \sigma_{t} \circ f_{t}^{T} \wedge d u_{T} \\
& =-\iint_{R_{t}} \sigma_{t} \wedge d\left(u_{T} \circ\left(f_{t}^{T}\right)^{-1}\right)=\int_{C_{t}} \sigma_{t}=\left\|\sigma_{t}\right\|_{R_{t}}^{2} .
\end{aligned}
$$

Thus Accola's Theorem ([1]) implies the assertion.

q.e.d.

Now $(d \lambda / d t)(T)$ exists for every $T$ by Lemma 4 and equals to $\operatorname{Re} \dot{I}(T)$, where 
$\dot{I}(T)$ is as in Lemma 4. Hence $\left(d^{2} \lambda / d t^{2}\right)(0)$ exists and (3) in Theorem 1 holds by Theorem 3 .

2. Proof of Theorem $1^{\prime}$. Set $\phi_{t}=-^{*} d g_{t}+i \cdot d g_{t}$ for every $t$, and let $\Phi^{T}(t-T)$ be defined as before. We recall one of standard definitions of Green's functions (cf. [3, Ch. IV, 6F]). For every $t$, take an exhaustion $\left\{S_{n}\right\}_{n=1}^{+\infty}$ of $R_{t}$ consisting of regular subregions $S_{n}$ with smooth boundary (cf. [3, Ch. II, 12D]). Assume that $p_{t} \in S_{1}$, and let $g_{t, n}$ be Green's function on $S_{n}$ with the pole $p_{t}$, i.e. the harmonic function on $S_{n}-\left\{p_{t}\right\}$ with singularity $-\log \left|Z_{t}\right|$ and boundary values 0 , for every $n$ (cf. [3, Ch. III, 15A]). Consider $g_{t, n}$ as a continuous function on $R_{t}$ by setting $g_{t, n} \equiv 0$ on $R_{t}-S_{n}$. Then it is well-known and easily seen that $g_{t}-g_{t, n}$ converges to 0 locally uniformly on $R_{t}$ as $n$ tends to $+\infty$, that $\left\|d\left(g_{t}-g_{t, n}\right)\right\|_{R_{t}}$ decreases as $n$ tends to $+\infty$, and that $\lim _{n \rightarrow+\infty}\left\|d\left(g_{t}-g_{t, n}\right)\right\|_{K}=0$ for every compact $K$ in $R_{t}$. Also we have the following

LEMma 7. The family $\left\{\Phi^{T}(s): s+T \in I\right\}$ satisfies $\left(\mathrm{A}^{T}\right),\left(\mathrm{B}^{T}\right)$ and $\left(\mathrm{C}^{T}\right)$ for every $T$.

Proof. Fix $T$ and $t$. First (iv) implies that $\Phi^{T}(t-T)$ is holomorphic in a neighborhood of $p_{T}$. And the same argument as in the proof of Lemma 5 shows that $\operatorname{Re} \Phi^{T}(t-T) \in \Gamma_{c}\left(R_{T}\right)$.

Next let $\left\{g_{t, n}\right\}_{n=1}^{+\infty}$ and $\left\{g_{T, n}\right\}_{n=1}^{+\infty}$ be as above, and set $h_{n}=g_{t, n} \circ f_{t}^{T}-g_{T, n}$ for every $n$. Then, since $\left\|d h_{n}-\operatorname{Im} \Phi^{T}(t-T)\right\|_{R_{T}} \leqq\left\|d\left(g_{t, n}-g_{t}\right) \circ f_{t}^{T}\right\|_{R_{T}}+\left\|d\left(g_{T, n}-g_{T}\right)\right\|_{R_{T}}$, $\left\{\left\|d h_{n}\right\|_{R_{T}}\right\}_{n=1}^{+\infty}$ is a bounded sequence and $\lim _{n \rightarrow+\infty}\left\|d h_{n}-\operatorname{Im} \Phi^{T}(t-T)\right\|_{K}=0$ for every compact set $K$. Hence it is easy to see (cf. [4, Hilfssatz 7.4]) that $d h_{n}$ converges weakly to $\operatorname{Im} \Phi^{T}(t-T)$ in $\Gamma\left(R_{t}\right)$, i.e. $\lim _{n \rightarrow+\infty}\left(d h_{n}, \omega\right)_{R_{T}}=$ $\left(\operatorname{Im} \Phi^{T}(t-T), \omega\right)_{R_{T}}$ for every $\omega \in \Gamma\left(R_{T}\right)$.

Now, since every $h_{n}$ has a compact support, Proposition 3 of [7] gives as before that $\left(d h_{n}, * \omega\right)_{R_{T}}=0$ for every smooth $\omega \in \Gamma_{c}\left(R_{T}\right)$. Hence $\left(\operatorname{Im} \Phi^{T}(t-T),{ }^{*} \omega\right)_{R_{T}}$ $=0$ for every such $\omega$. Thus we can conclude that $\operatorname{Im} \Phi^{T}(t-T) \in \Gamma_{e 0}\left(R_{T}\right)$.

Finally, since $\nu(t-T) \equiv 0$ on $f_{T}\left(U_{0}\right)$ for every $t$ (by (iv) and (8) in $\S 3$ ) and $\left\|d g_{T}\right\|_{R_{T}-U}$ is finite for every neighborhood $U$ of $p_{T}$, (B $\left.\mathrm{B}^{T}\right)$ holds with, for instance, $S_{T}=R_{T}-\left\{p \in R_{T}:\left|Z_{T}(p)\right| \leqq 1 / 2\right\}$. Also since $\Phi^{T}(t-T)$ is holomorphic on $f_{T}\left(U_{0}\right)$ and $p_{T}$ is the unique simple pole of $\phi_{T},\left(C^{T}\right)$ holds.

q.e.d.

LEMMA 8. For every $t$ and $T$,

$$
\gamma(t)-\gamma(T)=\frac{-1}{2 \pi} \cdot \operatorname{Re} \iint_{R_{T}} \Phi^{T}(t-T) \wedge * \phi_{T} .
$$

Proof (cf. the proof of Lemma 4 of [8]). Fix $t$ and $T$. Let $\left\{g_{T, n}\right\}$ be as before. Let $\chi(p)$ be a smooth function on $R_{T}$ with a compact support in $U_{0}$ such that $\chi(p) \equiv 1$ in a neighborhood of $p_{T}$, and set $\tilde{h}_{n}(p)=(1-\chi(p)) \cdot g_{T, n}(p)$ for every $n$. Then we can show as in the proof of Lemma 7 that $d \tilde{h}_{n}$ converges weakly to $(1-\chi) \cdot g_{T}$, and that $\left(d \tilde{h}_{n},{ }^{*} \omega\right)_{R_{T}}=0$ for every smooth $\omega \in \Gamma_{c}\left(R_{T}\right)$. Hence 
we conclude that $(1-\chi) \cdot g_{T} \in \Gamma_{e 0}\left(R_{T}\right)$. Since $\operatorname{Re} \Phi^{T}(t-T) \in \Gamma_{c}\left(R_{T}\right)$ by Lemma 7 , Green's formula gives

$$
\begin{aligned}
\iint_{R_{T}} \operatorname{Re} \Phi^{T}(t-T) \wedge d g_{T} & =\iint_{R_{T}} \operatorname{Re} \Phi^{T}(t-T) \wedge d\left(\chi \cdot g_{T}\right) \\
& =\lim _{\varepsilon \rightarrow 0} \int_{\partial U_{\varepsilon}} g_{T} \cdot \operatorname{Re} \Phi^{T}(t-T)=0,
\end{aligned}
$$

where we set $U_{\varepsilon}=\left\{p \in R_{T}:\left|Z_{T}(p)\right| \leqq \varepsilon\right\}$.

Thus again by Proposition 3 of $[7]$, we conclude that

$$
\begin{aligned}
\operatorname{Re} \iint_{R_{T}} \Phi^{T}(t-T) \wedge * \phi_{T} & =\iint_{R_{T}}-\operatorname{Im} \Phi^{T}(t-T) \wedge * d g_{T} \\
& =\lim _{\varepsilon \rightarrow 0}\left(\lim _{n \rightarrow+\infty} \int_{\partial U_{\varepsilon}} h_{n} * d g_{T}\right) \\
& =\lim _{n \rightarrow+\infty}-2 \pi \cdot h_{n}\left(p_{T}\right)=-2 \pi(\lambda(t)-\lambda(T)) . \quad \text { q.e.d. }
\end{aligned}
$$

Thus Lemma 4 and Theorem 3 give the assertion as in the last part of the proof of Theorem 1 .

Remark 3. If we used the notions of Royden's compactification and Dirichlet potentials (cf. [4]), then we could make some of above proofs geometrically clearer. But the author thinks the above proofs are reasonably elementary.

3. Proof of Theorem 2. In the sequel of this section, set $F(t)=\lambda(t)$ or $-2 \pi \cdot \gamma(t)$.

To prove Theorem 2, consider the 1-parameter families $\{\mu(t, 0): t \in \Re \cap U\}$ and $\{\mu(0, t): t \in \Re, \quad i t \in U\}$ with a real parameter $t$. Then $d / d t$ for these families correspond to $\partial / \partial x$ and $\partial / \partial y$, respectively. Hence by Theorems 1 and $1^{\prime}$, we have

$$
\begin{aligned}
\Delta F(0)= & \operatorname{Re} \iint_{R_{0}} \Delta \mu(0) \phi_{0} \wedge^{*} \phi_{0} \\
& +\operatorname{Re} \iint_{R_{0}}\left(\frac{\partial \Phi}{\partial x}(0) \wedge^{*} \frac{\partial \Phi}{\partial x}(0)+\frac{\partial \Phi}{\partial y}(0) \wedge^{*} \frac{\partial \Phi}{\partial y}(0)\right) .
\end{aligned}
$$

Since $\Delta \mu(0)=0$ by (v) and since

$$
\begin{aligned}
\iint_{R_{0}} \frac{\partial \Phi}{\partial x}(0) \wedge \frac{* \partial \Phi}{\partial y}(0) & =\left(\frac{\partial \Phi}{\partial x}(0), \frac{\overline{\partial \Phi}(0))_{R_{0}}^{\Subset}}{\partial y}\right. \\
& =\left(\frac{\partial \Phi}{\partial y}(0), \frac{\overline{\partial \Phi}(0)}{\partial x}\right)_{R_{0}}^{\Subset}=\iint_{R_{0}} \frac{\partial \Phi}{\partial y}(0) \wedge^{*} \frac{\partial \Phi}{\partial x}(0),
\end{aligned}
$$

we have 


$$
\Delta F(0)=4 \cdot \operatorname{Re} \iint_{R_{0}} \frac{\partial \Phi}{\partial \bar{t}}(0) \wedge^{*} \frac{\partial \Phi}{\partial t}(0) .
$$

Now set $\dot{\Phi}=(\partial \Phi / \partial \bar{t})(0)$. Since $\left(\partial \Phi^{0,1} / \partial \bar{t}\right)(0)=(\partial \mu / \partial \bar{t})(0) \cdot \phi_{0}=0$ by $(\mathrm{v})$ and $(4)$ in Lemma $1, \dot{\Phi}=\left(\partial \Phi^{1,0} / \partial \bar{t}\right)(0)$, and hence $* \dot{\Phi}=-i \cdot \dot{\Phi}$. Since $\dot{\Phi} \in \Gamma_{c}^{\circledR}\left(R_{0}\right)$ by $\left(\mathrm{A}^{0}\right), d \dot{\Phi}$ $=0$ and also $d^{*} \dot{\Phi}=-i \cdot d \dot{\Phi}=0$. Thus $\dot{\Phi} \in \Gamma_{h}^{\leftrightarrow s}\left(R_{0}\right)$ (, which implies further that $\dot{\Phi}$ is a holomorphic differential).

Since $\Psi=(\partial \Phi / \partial \bar{t})(0)-\overline{(\partial \Phi / \partial t)(0)}=2 i \cdot(\partial \operatorname{Im} \Phi / \partial \bar{t})(0) \in \Gamma_{e 0}^{\S}\left(R_{0}\right)$ by $\left(\mathrm{A}^{0}\right),(\dot{\Phi}, \Psi)_{R_{0}}^{\kappa}$ $=0$. Hence $\left(\dot{\Phi},(\overline{\partial \Phi / \partial t)(0)})_{R_{0}}^{\varsigma_{5}}=(\dot{\Phi}, \dot{\Phi})_{R_{0}}^{\kappa} \geqq 0\right.$. Thus we obtain $(4)$ and $\left(4^{\prime}\right)$ in Theorem 2 .

4. Proof of Corollary. First, continuity of $F(t)$ follows from Lemmas 6 and 8. In fact, fix $T=\left(T_{1}, \cdots, T_{n}\right)$. Since $\iint_{R_{T}} \Phi^{T}(t-T) \wedge^{*} \phi_{T}=\iint_{R_{T}}\left(\Phi^{T}\right)^{0,1}(t-T) \wedge^{*} \phi_{T}$, where $\left(\Phi^{T}\right)^{0,1}(t-T)$ is the projection of $\Phi^{T}(t-T)$ to $\Gamma^{0,1}\left(R_{T}\right)$, we can see by Lemma 4 that $|F(t)-F(T)|=O\left(\sum_{j=1}^{n}\left|t_{j}-T_{j}\right|\right)$ as $t$ tends to $T$.

Next fix a complex line $L=\left\{a t+b: t \in \mathbb{}, a \in \mho^{n}, b \in \mathbb{\mho}^{n}\right\}$ arbitrarily, and let $G$ be any component of $L \cap U$. Set $\Omega=\{t: a t+b \in G\}$ and $\mu^{L}(t)=\mu(a t+b)$ for every $t \in \Omega$. Then since $\mu^{L}(t)$ depends on $t$ holomorphically, $\left\{\mu^{L}(t)\right\}$ satisfies (ii') and $\partial \mu^{L} / \partial \bar{t} \equiv 0$ on $\Omega$. And since $\partial \mu^{L} / \partial t$ also depends on $t$ holomorphically, $\left\{\mu^{L}(t)\right\}$ satisfies (iii') and (v). Hence by Theorem $2, \Delta\left(\left.F\right|_{L}\right)(0)$ exists and nonnegative, where $\left.F\right|_{L}(t)=F(a t+b)$. Since $b$ can be taken arbitrarily in $G$, we conclude that $\Delta\left(\left.F\right|_{L}\right)$ exists and non-negative on the whole $\Omega$, which implies that $\left.F\right|_{L}$ is subharmonic on $\Omega$. (In fact, set $F_{\varepsilon}(t)=\left.F\right|_{L}(t)+\varepsilon \cdot|t|^{2}$ for every positive $\varepsilon$, and let $h_{\varepsilon}^{V}$ be the harmonic function on $V$ such that $h_{\varepsilon}^{V}=F_{\varepsilon}$ on $\partial V$ for every disk $V$ with $\bar{V} \subset \Omega$. If $\max _{\bar{V}}\left(h_{\varepsilon}^{V}-F_{\varepsilon}\right)$ were positive and hence were attained at a point $t_{0}$ in $V$, then $\Delta F_{\varepsilon}\left(t_{0}\right)$ should be non-positive, which contradicts to the fact that $\Delta F_{\varepsilon} \geqq 4 \cdot \varepsilon>0$. Hence $h_{\varepsilon}^{V} \leqq F_{\varepsilon}$ on $V$ for every $V$ as above. And letting $\varepsilon$ tend to 0 , we can conclude that $\left.F\right|_{L}$ is subharmonic on $\Omega$.) Since $L$ is arbitrary, we conclude the assertion.

\section{REFERENCES}

[1] R.D.M. Accola, Differentials and extremal length on Riemann surfaces, Proc. Nat. Acad. Sci. U.S. A., 46 (1960), 540-543.

[2] L. Ahlfors, Lectures on quasiconformal mappings, Van Nostrand, (1966), 146pp.

[3] L. Ahlfors and L. Sario, Riemann surfaces, Princeton, (1960), 382pp.

[4] C. Constantinescu And A. CoRnea, Ideale Ränder Riemannscher Flächen, Springer, (1963), 242pp.

[5] J. Dieudonne, Foundations of modern analysis, Pure and Applied Math. 10-I, Academic Press, (1969), 387pp.

[6] F. Maitani, Variations of meromorphic differentials under quasi-conformal deformations, J. Math. Kyoto Univ., 24 (1984), 49-66.

[7] C.D. MindA, Square integrable differentials on Riemann surfaces and quasicon- 
formal mappings, Trans. A.M.S., 195 (1974), 365-381.

[8] M. TANIGUCHI, A note on continuity of Green's functions on Riemann surfaces, Kodai Math. J., 10 (1987), 165-173.

[9] H. Yamaguchi, Calcul des variations analytiques, Japanese J. Math., 7 (1981). 319-377.

Department of Mathematics

KYOTO UNIVERSITY 